\section{Recombinase polymerase amplification: a promising point-of-care detection method for enteric viruses}

\author{
Matthew D Moore ${ }^{*, \neq, 1}$ \& Lee-Ann Jaykus ${ }^{1}$
}

Viral enteric disease imposes a considerable public health and economic burden globally in both humans and livestock. Because enteric viruses are highly transmissible and resistant to numerous control strategies, making early in-field or point-of-care detection is important. There are problems with ligand-based detection strategies (e.g., sensitivity, false positive/ negatives) for virus detection. Traditional amplification-based strategies are sensitive, but not as portable or rapid. Recombinase polymerase amplification is a new isothermal technique that utilizes bacterial genome repair enzymes to rapidly amplify target sequences. This report reviews the use of recombinase polymerase amplification for virus detection, showing that the method has favorable fundamental properties supporting its promise for rapid point-of-care detection of enteric viruses.

First draft submitted: 9 March 2017; Accepted for publication: 18 May 2017;

Published online: 21 June 2016

Enteric viral diseases impose a major public health and economic burden worldwide. Human noroviruses, the leading cause of acute viral gastroenteritis globally, were estimated to cause about 685 million infections and 212,000 deaths globally in 2010 [1]. Young children are more likely to become infected with enteric viruses, and the elderly and infirm are more likely to experience severe disease. Even though vastly improved with recent vaccination campaigns, WHO estimated that 215,000 children under the age of 5 died in 2013 from rotavirus infection [2]. Collectively, norovirus, rotavirus, hepatitis $\mathrm{A}$ and hepatitis $\mathrm{E}$ enteric viruses were estimated to have killed over 620,000 people globally in $2010[1,3]$. Other enteric viruses like astrovirus, sapovirus and adenovirus have been estimated to also inflict a notable public health burden [4-6]. Enteric viruses infecting livestock also inflict significant damage. For instance, the recently identified and highly contagious porcine epidemic diarrhea virus [7] has already caused devastating outbreaks with high animal mortality [8].

Because of factors like high transmissibility, asymptomatic shedding and relatively low infectious dose, the availability of early, portable detection of enteric viruses would considerably aid control and allow more timely measures to be taken to reduce transmission of enteric viruses, especially in long-term care, hospital and other institutional settings where highly transmissible viral outbreaks can be devastating [9-12]. Additionally, rapid point-of-care identification may also lower the unnecessary use of antibiotics, as antibiotic resistance is a growing concern [13]. Currently, the most widely used clinical detection assays that achieve some degree of favorable time-to-result and portability rely on ligand (i.e., antibody) binding. These ligand-based methods tend to have poorer sensitivity and an elevated potential of producing false negative and false positive results

'Department of Food, Bioprocessing \& Nutrition Sciences, North Carolina State University, Raleigh, NC 27695, USA

‡ Current Address: Enteric Diseases Laboratory Branch, Centers for Disease Control \& Prevention, 1600 Clifton Rd, Atlanta, GA 30329, USA *Author for correspondence: Tel.: +1 404718 3188; nni2@cdc.gov

\section{KEYWORDS}

- detection • enteric virus

- in-field • point-of-care

- recombinase polymerase amplification 
for multiple enteric viruses, especially norovirus and adenovirus; however, multiple successful, comparable commercial ligand-based tests exist for rotavirus [14-19]. Quantitative PCR (qPCR), an amplification-based method of detection, has a lower detection limit and greater inclusivity (ability to detect multiple virus strains) and hence is more sensitive than ligand-based methods $[14,20]$. But qPCR-based assays for viral detection are limited by their requirement for bulky instrumentation (for thermal cycling), sensitivity to sample matrix inhibitors and comparatively longer time-to-result. Because of this, multiple isothermal methods have been developed to remove the need of thermal cycling equipment. Perhaps the best known are loopmediated isothermal amplification (LAMP) and nucleic acid sequence-based amplification (NASBA). However, both require a large number of reagents/primers and generally take at least $1 \mathrm{~h}$ to complete. The purpose of this review is to present the recombinase polymerase amplification (RPA) assays developed for animal viruses with a focus on enteric viruses and describe a few of the reported properties of RPA that make it a promising technique for detection of enteric viruses. In depth discussion of LAMP and NASBA methods is beyond the scope of this review, but the interested reader is referred to more comprehensive papers [14,21,22].

Recombinase polymerase amplification RPA was first reported in 2006 [23] and represents one of the fastest isothermal amplification methods to date. All RPA kits are currently produced and under the intellectual license of TwistDx Limited (Cambridge, UK). The method relies on multiple bacterial proteins to amplify target DNA (Figure 1). Instead of heating and annealing, RPA utilizes a bacterial recombinase and single-stranded DNA binding protein to match primers to their target on the template DNA. A Staphylococcus aureus (Sau) polymerase then extends the primer and displaces the native strand. Additional co-factors are included in the reaction to aid the action of the enzymes (i.e., helper proteins, polyethylene glycol and ATP). A reverse transcription step can be included to make an RT-RPA assay capable of detecting RNA [24].

One of the advantages of the RPA assay is that the reaction can be conducted in a single tube and result in 'real-time' results. This is made possible by the inclusion of a fluorescent probe, of which there are three types for RPA reactions: exo, nfo and fpg. The exo probe (generally around 50 bases) contains an internal fluorophore and quencher separated by a tetrahydrofuran residue, which is the target of cleavage by exonuclease III only upon binding to the template DNA. Additionally, the probe contains a blocked 3' end to avoid its extension by the polymerase [24]. In a similar manner, the nfo probe also contains a tetrahydrofuran residue positioned in between a fluorophore and quencher. However, it is specifically recognized and cleaved by endonuclease IV (also called Nfo). This unblocks the $3^{\prime}$ end of the probe and it can be extended by a polymerase to eventually join a segment amplified by a primer with $5^{\prime}$ biotinylation. The resulting amplicon will then be labeled with biotin and a fluorophore, which allows for easy application on a lateral flow strip [23]. The fpg probe (generally $30-35$ bases) contains a $5^{\prime}$ quencher that is a few bases away from an abasic deoxyribose to which a fluorophore is linked. A nuclease (Fpg) specifically recognizes and cuts the linked fluorophore when the probe is annealed [24]. Traditionally, RPA primers were recommended to be $30-35$ bases in length; however TwistDx has recently announced that PCR primers (18 bases and higher) can successfully be used [25].

\section{RPA for detection of viruses}

Numerous RPA assays have been successfully developed and reported for both RNA and DNA viruses (Table 1). Some RT-RPA assays have been developed for enteric viruses including bovine coronavirus, bovine viral diarrhea virus (BVDV) and human norovirus. Amer et al. [26] reported the development of an assay for bovine coronavirus that had slightly better analytical sensitivity $\left(10^{1}-10^{2}\right.$ molecules/ $\left.\mu \mathrm{l}\right)$ than RT-qPCR $\left(10^{3}\right.$ molecules $/ \mu \mathrm{l})$. The time to threshold fluorescence correlated with RT-qPCR Ct values and the assay did not amplify bovine rotaviruses, BVDV, infectious bovine rhinotracheitis, bovine parainfluenza type 3 and bovine respiratory syncytial virus. Further, the assay performed well for detecting bovine coronavirus from clinical nasal and fecal samples [26]. Aebischer et al. [27] developed and compared RT-qPCR, LAMP and RT-RPA assays for two bovine enteric viruses, Schmallenberg virus and BVDV. The RT-RPA assay consistently outperformed the other two assays with regard to time-to-result and portability but had a slightly higher detection limit than RT-qPCR and LAMP assays. Nonetheless, the 
(A) Recombinase/oligonucleotide primer complexes form and target homogenous DNA

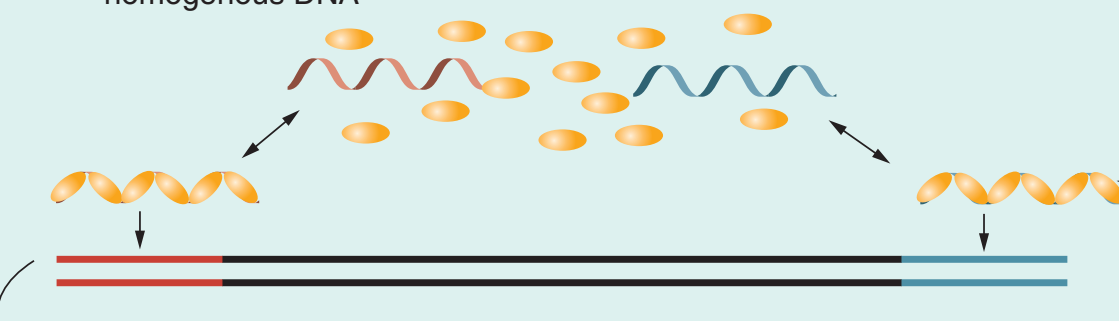

(B) Strand exchange forms a D-loop
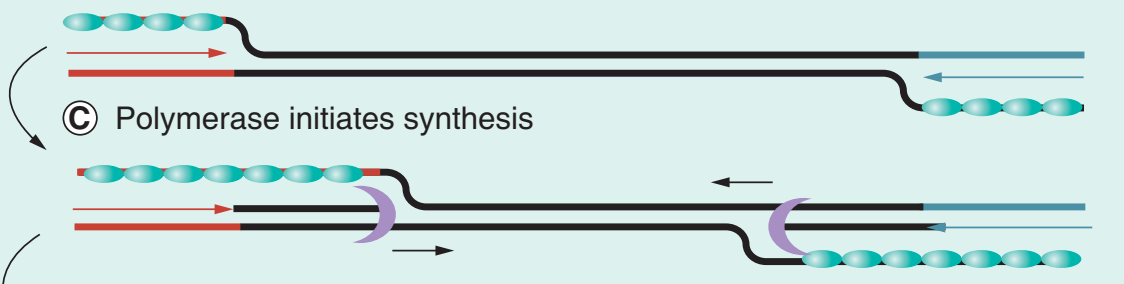

(D) Parental strands separate and synthesis continues

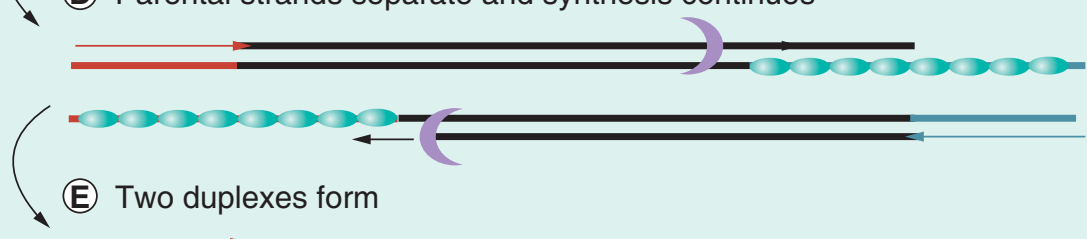

$+$

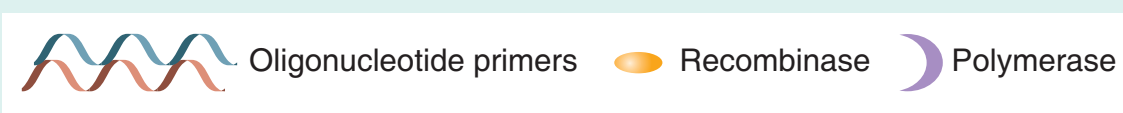

\section{SSB}

Figure 1. Figure schematic of the recombinase polymerase amplification process. A layout of the steps involved in RPA amplification is provided. First, recombinases bind primers pair to complimentary sequence on template DNA. With the aid of single-strand binding protein to bind exposed DNA, the primer is paired and a D-loop is formed. A polymerase extends the primer, eventually displacing the two original strands of template DNA. This process then repeats for amplification.

RPA: Recombinase polymerase amplification.

Figure courtesy TwistDx Limited. Schematics of the mechanisms of different probes are not pictured.

limit of detection for the RT-RPA was below that needed for detection of the virus in clinical samples from animals exhibiting symptomatic infection [27]. Moore and Jaykus [28] developed and compared an RT-RPA assay for the detection of epidemic human norovirus (GII.4 New Orleans) with a traditional RT-qPCR assay. As in the previous study, the RT-RPA assay had a slightly higher limit of detection $\left(\sim 1 \log _{10}\right.$ genomic copy/ reaction), but was capable of detecting human norovirus in RNA extracts of all clinical stool samples tested in less than 20 min compared with $2 \mathrm{~h}$ required for RT-qPCR [28]. Numerous other viral RPA assays have been developed and are summarized in Table 1.

\section{Advantages \& challenges of RPA for enteric virus detection \\ - Analytical sensitivity (limit of detection)}

As described above, there have been mixed results regarding the comparability of RT-RPA analytical sensitivity with other nucleic acid amplification-based detection methods. In some cases, RPA demonstrates detection limits com- 
parable or even better than those achieved by RT-qPCR [26,29-32] (Table 1). However, in other reports, viral RPA assays had a slightly higher limit of detection and hence poorer diagnostic sensitivity. This could be due to multiple factors including the methodology used to determine assay detection limit. For example, Euler et al. [29] developed an RT-RPA assay for Rift Valley fever virus with a measured analytical sensitivity of 19 molecules using serial dilutions of an RNA standard in a clean solution with a relevant segment of the viral genome in a plasmid used as template. However, this scenario would be considered a best-case scenario for determining detection limits, as it does not account for the effect of matrix-associated components, such as unrelated genomic material that would be competing for recombinase enzymes or inhibitory sample components. Abd el Wahed et al. [33] illustrated this principle when they demonstrated a $1-2 \log _{10}$ improvement in detection limit of their RT-RPA assay for dengue virus when using an RNA stand- ard as compared with heat-treated virus spiked into serum. Similar results were observed by Moore et al. [28] for human norovirus. Amplicon length may also influence sensitivity, as assays with shorter amplicons have been among the most sensitive $[26,29,34]$. This may potentially be due to a lower number of enzymes (like singlestrand binding protein) required per amplicon; however, further study of the effects of amplicon length is needed. Additionally, the quality and degree of screening involved in designing the primers for these assays likely has an effect on sensitivity, as primer refinement is a continuous process. Regardless, it should be noted that even the RPA assays with lower analytical sensitivity still generally have relevance and promise for detection in clinical samples where virus loads are usually quite high. As these RPA assays are quite sensitive, application of these for the purpose of clinical diagnosis should account for the possibility of co-infection or asymptomatic infection of the detected pathogen. As with other sensitive molecular methods, care should

Table 1. Recombinase polymerase amplification assays for detection of animal viruses.

\begin{tabular}{|c|c|c|c|}
\hline Virus & Sample matrix ${ }^{\dagger}$ & Analytical sensitivity & Ref. \\
\hline \multirow[t]{2}{*}{ Dengue virus } & Serum & $11 \mathrm{GC}$ & [35] \\
\hline & Serum & $241(1-3)^{\ddagger}, 14(4) \mathrm{GC}$ & [33] \\
\hline \multirow[t]{4}{*}{ HIV } & $\begin{array}{l}\text { Plasmid DNA supplemented with } \\
\text { human DNA }\end{array}$ & $\sim 10 \mathrm{GC}$ & {$[42]$} \\
\hline & Blood/cell line & $1-10 \mathrm{GC}$ & [36] \\
\hline & Plasmid DNA & $10 \mathrm{GC}$ & {$[44]$} \\
\hline & Blood/cell line & $10 G C^{\S}$ & {$[36,54]$} \\
\hline Influenza H7N9 (avian) & Transcribed RNAs & $10(\mathrm{H} 7), 100$ (N9) GC & [31] \\
\hline $\begin{array}{l}\text { Infectious hypodermal and hematopoietic } \\
\text { necrosis virus }\end{array}$ & Shrimp and crab hepatopancreas & $4 \mathrm{GC}$ & {$[30]$} \\
\hline Bovine coronavirus & Cell line extract, fecal/nasal swabs & $19 \mathrm{GC}$ & [26] \\
\hline Schmallenberg virus, bovine viral diarrhea virus & Blood/serum & $6 \times 10^{3}(\mathrm{SBV}), 5 \times 10^{4}(\mathrm{BVDV}) \mathrm{GC}$ & [27] \\
\hline Middle East respiratory syndrome coronavirus & Transcribed RNAs & $21 \mathrm{GC}$ & [48] \\
\hline Rift Valley fever virus & Transcribed RNAs & $19 \mathrm{GC}$ & [29] \\
\hline $\begin{array}{l}\text { Rift Valley fever virus, variola virus, Ebola virus, } \\
\text { Sudan virus, Marburg virus and Sigma virus }\end{array}$ & Plasma & $\begin{array}{l}19 \text { (RVFV), } 16 \text { (Var), } 21 \text { (Ebola), } 17 \text { (Sudan), } \\
21 \text { (Mar), } 16 \text { (Sig) GC }\end{array}$ & \\
\hline Yellow fever virus & $\begin{array}{l}\text { Cell culture supernatant, mosquito, } \\
\text { spiked human plasma }\end{array}$ & $21-44$ GC & {$[55]$} \\
\hline Human norovirus & Stool & $2.51 \times 10^{3} \mathrm{GC}$ & [28] \\
\hline Feline herpesvirus 1 & Nasal and ocular conjunctival swabs & $10^{2} \mathrm{GC}$ & [32] \\
\hline White spot syndrome virus & Shrimp & $10 \mathrm{GC}$ & [56] \\
\hline \multicolumn{4}{|c|}{$\begin{array}{l}\text { †Analytical sensitivity was, in most cases, determined using RNA/DNA standards produced from purified plasmids or transcribed RNA (cDNA). Although application to clinical } \\
\text { samples from relevant sample matrices may have been applied as proof-of-concept, naturally contaminated samples were rarely used to determine analytical sensitivity. } \\
\text { "Detection limit was determined for dengue virus serotypes } 1-3 \text { using a pure RNA standard. } \\
\text { sMust be at temperatures of } 30^{\circ} \mathrm{C} \text { or greater. }\end{array}$} \\
\hline
\end{tabular}


be taken to test for multiple agents before utilizing RPA for diagnosis. Overall, the favorable sensitivity of RPA makes it promising not just for clinical detection, but for detection of enteric viruses in food, water and environmental samples as well.

\section{- Fidelity}

One of the prime advantages cited for RPA is the potentially higher degree of fidelity of the assay, which can result in a test of high specificity. This is not surprising, as the assay inherently relies upon proofreading enzymes for amplification. In the majority of the assays, specificity appears comparable or better than RT-qPCR when a comparison is made [27,35], and the RPAs reported to date demonstrate negative results when panels of related viruses have been directly tested. Although such specificity is desirable in detection of bacterial and eukaryotic pathogens that contain longer genomes and are generally more conserved, it may be less attractive in the case of virus detection. This is because some viruses (e.g., noroviruses and enteroviruses) have comparably small genomes and can display genetic diversity making it difficult to identify primers and probes that cross-react with the diversity of strains within a specific virus genus or family $[27,28]$. This is partially attributed to the longer primer and probe length for RPA relative to $\mathrm{qPCR}$; however, as stated above, TwistDx has recently reported on the successful use of shorter primers [25]. Despite this high specificity, RPA has been widely documented to tolerate some degree of base mismatch [28,36,37]. Specifically, Daher et al. [37] generated 87 different mismatched versions of forward primers for a panel of bacteria and report observing that negative effects can occur more frequently with location of mismatch ( $3^{\prime}$ extremity) as well as number of mismatches ( $>3$, especially located near extremities). Similar anecdotal observation related to viruses has been reported [28], but a more empirical study needs to be performed for viruses, especially those with RNA genomes.

\section{- Toleration of matrix-associated inhibitors} One of the major hindrances to rapid pointof-care testing is the requirement for efficient sample preparation prior to detection. This includes volume reduction, removal of components of the sample matrix and genome purification. RT-qPCR is particularly susceptible to inhibitors, therefore identifying rapid assays with better tolerance is important. Studies have shown variations in the tolerance of different isothermal techniques to matrix-associated inhibitors [38-40]. Multiple reports suggest that RPA may better tolerate matrix inhibition than RT-qPCR. For example, Krôlov et al. [41] were able to detect Chlamydia trachomatis directly from heat-treated urine, which contains PCR inhibitors. Similarly, Abd el Wahed et al. [33] successfully demonstrated limits of detection of $10^{2}-10^{3}$ RNA molecules for inactivated dengue virus spiked into serum, diluted, and directly used for RT-RPA. Moore and Jaykus [28] successfully detected human norovirus from patient stool that was simply diluted, heated and used as template for RT-RPA. Additionally, the RT-RPA assay performed better than an RT-qPCR assay at higher concentrations of heated stool [28]. However, sensitivity to inhibition at very low template concentrations has still been observed for RPA. This may be less of a problem for clinical samples having high target concentrations. The use of internal controls can help identify inhibition when it occurs, thereby reducing the likelihood of false negative results [42].

\section{- Portability \& simplicity of preparation}

The lack of a need for bulky instrumentation is one of the major advantages of RPA for pointof-care detection of enteric viruses. All that is required for detection beyond the assay reagents and pipettes is a battery-powered device the size of a small textbook. This advantage is best exemplified in an assay developed for avian influenza H7N9, in which RPA was entirely performed with equipment that fits into a $56 \mathrm{~cm} \times 45 \mathrm{~cm} \times$ $26.5 \mathrm{~cm}$ suitcase. The assay performed with this unit showed analytical sensitivity comparable to PCR, needed only a solar-powered battery for operation, and did not require a cold chain [31]. In the same manner, an HIV-1 RPA assay was reported where incubation was performed with body heat [43]. Additionally, the ease with which RPA reactions are prepared is an advantage for point-of-care testing, as RPA enzymes are provided in lyophilized pellets, thus only buffer, template, primers and probe need to be added. This is especially favorable compared with other isothermal assays that require a diverse set of primers, longer incubation times and higher incubation temperatures. Additionally, this allows for more flexible storage/transport conditions. Furthermore, work to miniaturize RPA assays onto chips has been conducted [44-46], 
making potential RPA portability and ease of preparation for point-of-care testing even more promising.

\section{- Time-to-result}

Perhaps one of the biggest advantages of the RPA method is the rapid time-to-result, as the entire reaction takes no more than 20-30 min. Achieving results in less than $10 \mathrm{~min}$ is fairly common, especially in clinical samples having high virus titers; this has been observed in numerous studies [26,28,31,47-49]. In short, RPA routinely displays faster time-to-result than other amplification methods [27]. Traditional real-time PCR, LAMP and NASBA assays take $60 \mathrm{~min}$ or more. Aebischer et al. [27] optimized enteric virus real-time PCR and LAMP assays to $30 \mathrm{~min}$; however, RPA provided the fastest time to signal compared with the other methods for all of the RPA-positive samples.

\section{- Cost}

As with any point-of-care or in-field detection method, cost is a major consideration. The cost of the RPA assay relative to other assays will depend upon each unique situation in which it is being applied. Factors affecting the relative expense of RPA to other assays include the pathogen, requirement for equipment, infrastructure/cold storage capacity, degree of employee hourly expense, volume of samples tested and sample matrix being tested, among others. Zhang et al. [50] estimated the cost of an RPA platform for plum pox virus as US $\$ 30$ or less per sample, and suggested it was likely more cost effective than traditional methods for plum pox virus like PCR or immunoassay based on the reduced time, labor and preparation/transportation required for the latter tests compared with the RPA. The extent of sample preparation is a major factor, as nucleic acid isolation of little cherry virus 2 for RT-PCR was estimated to comprise about half of the cost of analysis. This was largely due to the ability of the RPA to perform favorably when crude extracts were directly analyzed and nucleic acid extraction was not needed [51]. In addition to potentially reducing costs by obviating the need for nucleic acid purification, Teoh et al. [35] noted that the real-time fluorometer required for RPA assays was four-times less expensive than the price for an LAMP device and six-times lower than devices required for PCR. Because the optimal incubation temperature of RPA assays is around $40^{\circ} \mathrm{C}$, Crannell et al. [43] reduced costs and the requirement for electricity by developing a bodyheat-based system for incubating RPA reactions that would be measured in an established lateral flow detection format for HIV. Although in these respects RPA reduces costs, there are some challenges. Namely, reagent costs for RPA are quite high compared with real-time PCR and immune-based assays, especially with regard to the fluorescent probes used for real-time detection. Further, the reactions generally are available in eight reaction strips, limiting throughput. However, future work and innovation involving multiplexing RPA and creating lab-on-a-chip capable of multiple simultaneous RPA assays is already underway [46,52].

\section{Future perspective}

RPA is one of the newer isothermal amplification strategies, and its application is likely to expand due to its numerous advantages including rapid time to result, portability, fidelity, simplicity and sensitivity. Only a few enteric virus RPA assays have been reported to date [26-28], and the authors suspect that existing assays will be improved as more is understood about the specific nature of RPA, especially regarding analytical sensitivity and cross-reactivity. Specifically, work is needed to empirically determine the suitability of shorter, more degenerate RPA primers for enteric virus detection; the mechanisms and degree of base mismatch tolerated by RT-RPA; the effects of template's secondary structure on RPA assay performance; further elucidating the most favorable primer sequence features; the development of multiplexed RPA assays; and further characterization of the effect of amplicon length on RPA sensitivity. There is clearly a need to develop new RPA assays for other highly transmissible enteric viruses. The authors anticipate that such development will occur in the coming years, and the method will be increasingly applied to virus detection in clinical samples, and perhaps for foods and environmental samples. Future work involving integrating RPA assays into lab-on-a-chip [45] and handheld devices [53] for enteric virus detection is also likely to occur.

\section{Conclusion}

In conclusion, RPA is a promising method for point-of-care and in-field detection of enteric viruses and deserves to be further developed in upcoming years. 
Acknowledgements

We would like to thank HC Bee for her opinion on the readability of this manuscript.

\section{Financial \& competing interests disclosure}

This review was prepared with partial support from the National Institute of Food and Agriculture, US Department of Agriculture, award number 1111-2011-0494, under the NoroCORE project. The authors have no ties with TwistDx Limited beyond obtaining permission to use their figure for the schematic of the RPA assay, and declare no conflicts of interest. The authors have no other relevant affliations or financial involvement with any organization or entity with a financial interest in or financial conflict with the subject matter or materials discussed in the manuscript apart from those disclosed.

No writing assistance was utilized in the production of this manuscript.

\section{Open access}

This work is licensed under the Attribution-NonCommercial-NoDerivatives 4.0 Unported License. To view a copy of this license, visit http://creativecommons.org/ licenses/by-nc-nd/4.0/

\section{EXECUTIVE SUMMARY}

- Enteric viral diseases are a major public health and economic concern. Because of transmissibility, early detection of viral infections in-field or at point-of-care is important for control.

- Ligand-based detection (e.g., ELISA) methods are fast and portable, but lack sensitivity and can cross-react to produce false positive results.

- Quantitative PCR is sensitive and specific, but is not portable and generally takes $>1.5 \mathrm{~h}$.

- Recombinase polymerase amplification (RPA) utilizes bacterial enzymes to isothermally amplify target nucleic acids in under 20 min.

- RPA uses bacterial recombinases as well as single-stranded binding protein and other co-factors to anneal primers to a target sequence for extension/amplification by a polymerase.

- Numerous RPA methods for detection of viruses have been reported.

- Enteric virus RPA assays have been developed for bovine coronavirus, bovine viral diarrhea virus, Schmallenberg virus, and human norovirus.

- Reports are mixed regarding the sensitivity of RPA relative to PCR methods - many reports show RPA has comparable or better analytical sensitivity than PCR while some show RPA to be less sensitive than PCR.

- RPA has been shown to exhibit high fidelity in amplification of target sequence with good clinical specificity.

- Emerging evidence suggests RPA may be more tolerant to sample matrix-associated inhibitors than PCR.

- Because of its isothermal nature, RPA has been utilized in a portable manner, including on chips and lateral flow strips.

- RPA displays among the fastest time-to-result of all available nucleic acid amplification techniques.

- The RPA method has numerous advantages that make it a promising method for point-of-care detection of enteric viruses.

\section{References}

Papers of special note have been highlighted as:

- of interest; $\bullet$ of considerable interest

1 Kirk MD, Pires SM, Black RE et al. World Health Organization estimates of the global and regional disease burden of 11 foodborne bacterial, protozoal, and viral diseases, 2010: a data synthesis. PLoS Med. 12(12), e1001920 (2015).

2 World Health Organization. Estimated rotavirus deaths for children under 5 years of age: 2013.215000 (2017)
3 Lozano R, Naghavi M, Foreman K et al. Global and regional mortality from 235 causes of death for 20 age groups in 1990 and 2010: a systematic analysis for the Global Burden of Disease Study. Lancet 380(9859), 2095-2128 (2012).

4 Tam CC, Rodrigues LC, Viviani L et al. Longitudinal study of infectious intestinal disease in the UK (IID2 study): incidence in the community and presenting to general practice. Gut 61(1), 69-77 (2012).
5 Bosch A, Pinto RM, Guix S. Human astroviruses. Clin. Microbiol. Rev. 27(4), 1048-1074 (2014).

6 Platts-Mills JA, Babji S, Bodhidatta L et al. Pathogen-specific burdens of community diarrhoea in developing countries: a multisite birth cohort study (MAL-ED). Lancet Glob. Health 3(9), 564-575 (2015).

7 Song D, Park B. Porcine epidemic diarrhoea virus: a comprehensive review of molecular epidemiology, diagnosis, and vaccines. Virus Genes. 44(2), 167-175 (2012). 
8 Chen Q, Li G, Stasko J et al. Isolation and characterization of porcine epidemic diarrhea viruses associated with the 2013 disease outbreak among swine in the United States. J. Clin. Microbiol. 52(1), 234-243 (2014).

9 Lee BY, McGlone SM, Bailey RR, Wettstein ZS, Umscheid CA, Muder RR. Economic impact of outbreaks of norovirus infection in hospitals. Infect. Control Hosp. Epidemiol. 32(2), 191-193 (2011).

10 Koopmans M. Noroviruses in healthcare settings: a challenging problem. J. Hosp. Infect. 73(4), 331-337 (2009).

11 Danial J, Cepeda Ja, Cameron F, Cloy K, Wishart D, Templeton KE. Epidemiology and costs associated with norovirus outbreaks in NHS Lothian, Scotland 2007. J. Hosp. Infect. 79(4), 354-358 (2011).

12 Lee BY, Wettstein ZS, McGlone SM et al. Economic value of norovirus outbreak control measures in healthcare settings. Clin. Microbiol. Infect. 17(4), 640-646 (2011).

13 Costelloe C, Metcalfe C, Lovering A, Mant D, Hay AD. Effect of antibiotic prescribing in primary care on antimicrobial resistance in individual patients: systematic review and meta-analysis. BMJ 340, c2096 (2010).

14 Law JW, Ab Mutalib NS, Chan KG, Lee LH. Rapid methods for the detection of foodborne bacterial pathogens: principles, applications, advantages and limitations. Front. Microbiol. 5, 770 (2015).

15 Rovida F, Campanini G, Sarasini A, Adzasehoun KMG, Piralla A, Baldanti F. Comparison of immunologic and molecular assays for the diagnosis of gastrointestinal viral infections. Diagn. Microbiol. Infect. Dis. 75(1), 110-111 (2013).

16 Ambert-Balay K, Pothier P. Evaluation of 4 immunochromatographic tests for rapid detection of norovirus in faecal samples. J. Clin. Virol. 56(3), 194-198 (2013).

17 Ye S, Roczo-Farkas S, Whiley DM et al. Evidence of false-positive results in a commercially available rotavirus assay in the vaccine era, Australia, 2011 to. Eurosurveillance 18(21), 20483 (2013).

18 Ye S, Lambert SB, Grimwood K et al. Comparison of test specificities of commercial antigen-based assays and in-house PCR methods for detection of rotavirus in stool specimens. J. Clin. Microbiol. 53(1), 295-297 (2015).

19 Dusetty P, Velázquez FR, Gutiérrez-Escolano AL, Ludert JE. Evaluation of the second generation of a commercial latex agglutination test for the detection of rotavirus antigens in fecal samples. J. Clin. Virol. 57(1), 88-90 (2013).

20 Zhao X, Lin CW, Wang J, Oh DH. Advances in rapid detection methods for foodborne pathogens. J. Microbiol. Biotechnol. 24(3), 297-312 (2014).

21 Mori Y, Notomi T. Loop-mediated isothermal amplification (LAMP): a rapid, accurate, and cost-effective diagnostic method for infectious diseases. J. Infect. Chemother. 15(2), 62-69 (2009).

22 Wang $\mathrm{H}, \mathrm{Mo}$ Q, Yang Z. RT-NASBA: an ideal approach for the detection of diarrhoearelated viruses. Br. J. Virol. 2(3), 37-42 (2015).

23 Piepenburg O, Williams CH, Stemple DL, Armes NA. DNA detection using recombination proteins. PLoS Biol. 4(7), e204 (2006).

- Original report describing recombinase polymerase amplification (RPA) in the peer-reviewed scientific literature.

24 Daher RK, Stewart G, Boissinot M, Bergeron MG. Recombinase polymerase amplification for diagnostic applications. Clin. Chem. 62(7), 947-958 (2016).

25 Twist Dx, Inc. PCR primers work using standard RPA reagents (2017).

26 Amer HM, Abd El Wahed A, Shalaby MA, Almajhdi FN, Hufert FT, Weidmann M. A new approach for diagnosis of bovine coronavirus using a reverse transcription recombinase polymerase amplification assay. J. Virol. Methods 193(2), 337-340 (2013).

- Describes an RT-RPA assay with high sensitivity for an enteric virus. The limit of detection is comparable or slightly better than RT-quantitative PCR.

27 Aebischer A, Wernike K, Hoffmann B, Beer M. Rapid genome detection of Schmallenberg virus and bovine viral diarrhea virus by use of isothermal amplification methods and high-speed real-time reverse transcriptase PCR. J. Clin. Microbiol. 52(6), 1883-1892 (2014).

- This is another report involving enteric viruses. It includes RPA methods in addition to loop-mediated isothermal amplification and PCR assays and compares them. In this case, the RPA assay developed was less sensitive than the other two but consistently outperformed the other two methods with respect to detection time.

28 Moore MD, Jaykus LA. Development of a recombinase polymerase amplification assay for detection of epidemic human noroviruses. Sci. Rep. 7, 40244 (2017).
- Reports the development of an RT-RPA assay for the epidemic strain of a human enteric virus and compares it with RT-qPCR. The RT-RPA assay is consistently faster and some evidence is presented where it is more tolerant of inhibitors from stool; however, it is less sensitive than RT-qPCR.

29 Euler M, Wang Y, Nentwich O, Piepenburg O, Hufert FT, Weidmann M. Recombinase polymerase amplification assay for rapid detection of Rift Valley fever virus. J. Clin. Virol. 54(4), 308-312 (2012).

30 Xia X, Yu Y, Hu L et al. Rapid detection of infectious hypodermal and hematopoietic necrosis virus (IHHNV) by real-time, isothermal recombinase polymerase amplification assay. Arch. Virol. 160(4), 987-994 (2015).

31 Abd El Wahed A, Weidmann M, Hufert FT. Diagnostics-in-a-Suitcase: development of a portable and rapid assay for the detection of the emerging avian influenza $\mathrm{A}(\mathrm{H} 7 \mathrm{~N} 9)$ virus. J. Clin. Virol. 69, 16-21 (2015).

- Demonstrates the potential portability of RPA, as every material needed for detection of avian influenza from sample-to-signal is included in a suitcase. Electricity is not needed.

32 Wang J, Liu L, Wang J, Sun X, Yuan W. Recombinase polymerase amplification assay - a simple, fast and cost-effective alternative to real time PCR for Specific detection of feline herpesvirus. PLoS ONE 12(1), e0166903 (2017).

33 Abd El Wahed A, Patel P, Faye O et al. Recombinase polymerase amplification assay for rapid diagnostics of dengue infection. PLoS ONE 10(6), 1-17 (2015).

34 Euler M, Wang Y, Otto P et al. Recombinase polymerase amplification assay for rapid detection of Francisella tularensis. J. Clin. Microbiol. 50 (7), 2234-2238 (2012).

35 Teoh BT, Sam SS, Tan KK et al. Early detection of dengue virus by use of reverse transcription-recombinase polymerase amplification. J. Clin. Microbiol. 53(3), 830-837 (2015).

36 Boyle DS, Lehman DA, Lillis L. Rapid detection of HIV-1 proviral DNA for early infant diagnosis using recombinase polymerase amplification. MBio 4(2), e00135-e00113 (2013).

37 Daher RK, Stewart G, Boissinot M, Boudreau DK, Bergeron MG. Influence of sequence mismatches on the specificity of recombinase polymerase amplification technology. Mol. Cell. Probes 29(2), 116-121 (2015). 
T, Fukushima E, Suzutani T. Tolerance of loop-mediated isothermal amplification to a culture medium and biological substances. $J$. Biochem. Biophys. Methods 70(3), 499-501 (2007).

39 Greene SR, Moe CL, Jaykus LA, Cronin M, Grosso L, Aarle Pv. Evaluation of the NucliSens $s^{\circledR}$ Basic Kit assay for detection of Norwalk virus RNA in stool specimens. J. Virol. Methods. 108(1), 123-131 (2003).

40 Enomoto Y, Yoshikawa T, Ihira M et al. Rapid diagnosis of herpes simplex virus infection by a loop-mediated isothermal amplification method. J. Clin. Microbiol. 43(2), 951-955 (2005).

41 Krôlov K, Frolova J, Tudoran O et al. Sensitive and rapid detection of Chlamydia trachomatis by recombinase polymerase amplification directly from urine samples. J. Mol. Diagnostics 16(1), 127-135 (2014).

42 Crannell ZA, Rohrman B, Richards-kortum R. Quantification of HIV-1 DNA using real-time recombinase polymerase amplification. Anal. Chem. 86, 5615-5619 (2014).

43 Crannell ZA, Rohrman B, Richards-Kortum R. Equipment-free incubation of recombinase polymerase amplification reactions using body heat. PLoS ONE 9(11), 1-7 (2014).

Rohrman BA, Richards-Kortum RR. A paper and plastic device for performing recombinase polymerase amplification of HIV DNA. Lab Chip 12(17), 3082 (2012).
45 Lutz S, Weber P, Focke M et al. Microfluidic lab-on-a-foil for nucleic acid analysis based on isothermal recombinase polymerase amplification (RPA). Lab Chip 10(7), 887-893 (2010).

46 Kersting S, Rausch V, Bier FF, von NickischRosenegk M. Multiplex isothermal solidphase recombinase polymerase amplification for the specific and fast DNA-based detection of three bacterial pathogens. Mikrochim. Acta 181(13-14), 1715-1723 (2014).

47 Euler M, Wang Y, Heidenreich D et al. Development of a panel of recombinase polymerase amplification assays for detection of biothreat agents. J. Clin. Microbiol. 51(4), 1110-1117 (2013).

Abd El Wahed A, Patel P, Heidenreich D, Hufert FT, Weidmann M. Reverse transcription recombinase polymerase amplification assay for the detection of Middle East respiratory syndrome coronavirus. PLoS Curr. doi:10.1371/currents. outbreaks.62df1c7c75ffc96cd59034531e $2 \mathrm{e} 8364$ (2013).

\section{Abd El Wahed A, El-Deeb A, El-Tholoth M} et al. A portable reverse transcription recombinase polymerase amplification assay for rapid detection of foot-and-mouth disease virus. PLoS ONE 8(8), 1-7 (2013).

Zhang S, Ravelonandro M, Russell P et al. Rapid diagnostic detection of plum pox virus in Prunus plants by isothermal AmplifyRP ${ }^{\circledR}$ using reverse transcription-recombinase polymerase amplification. J. Virol. Methods
207, 114-120 (2014).

51 Mekuria TA, Zhang S, Eastwell KC. Rapid and sensitive detection of Little cherry virus 2 using isothermal reverse transcriptionrecombinase polymerase amplification. J. Virol. Methods 205, 24-30 (2014).

52 Shen F, Davydova EK, Du W, Krutz JE, Piepenburg O, Ismagilov RF. Digital isothermal quantification of nucleic acids via simultaneous chemical initiation of recombinase polymerase amplification reactions on SlipChip. Anal. Chem. 83(9), 3533-3540 (2011).

53 Selck DA, Karymov MA, Sun B, Ismagilov RF. Increased robustness of single-molecule counting with microfluidics, digital isothermal amplification, and a mobile phone versus real-time kinetic measurements. Anal. Chem. 85(22), 11129-11136 (2013).

54 Lillis L, Lehman D, Singhal MC et al. Non-instrumented incubation of a recombinase polymerase amplification assay for the rapid and sensitive detection of proviral HIV-1 DNA. PLoS ONE 9(9), 6-9 (2014).

55 Escadafal C, Faye O, Sall AA et al. Rapid molecular assays for the detection of yellow fever virus in low-resource settings. PLoS Negl. Trop. Dis. 8(3), (e2730) (2014).

56 Xia X, Yu Y, Weidmann M, Pan Y, Yan S, Wang Y. Rapid detection of shrimp white spot syndrome virus by real time, isothermal recombinase polymerase amplification assay. PLoS ONE 9(8), 1-8 (2014). 